\title{
Upper-limb exoskeletons for stroke rehabilitation
}

\author{
Emilia Ambrosini ${ }^{1}$, Stefano Dalla Gasperina ${ }^{1}$, Marta Gandolla ${ }^{2}$, and \\ Alessandra Pedrocchi ${ }^{1}$ \\ 1 NearLab, Department of Electronics, Information and Bioengineering, Politecnico \\ di Milano, Italy \\ 2 NearLab@Lecco, Polo Territoriale di Lecco, Politecnico di Milano, Italy \\ emilia.ambrosini@polimi.it
}

\begin{abstract}
Upper-limb exoskeletons provide high-intensity, repetitive, task-specific, interactive and individualized training, making effective use of neuroplasticity for functional recovery in neurological patients. Most exoskeletons have robot axes aligned with the anatomical axes of the subject and provide direct control of individual joints. Recently, novel mechanical structures and actuation mechanisms have been proposed, but still result in bulky and heavy exoskeletons, limiting their applicability into clinical practice. Technological efforts are needed to promote light and wearahle exoskeletons that implement active-assistive controllers, providing ass isted-as-needed" rehabilitation therapy, towards patient's motivation and self-esteem. An overview of upper-limb exoskeletons, including mechanical design and control algorithms, will be provided. Special focus will be put on the current evidence about the efficacy of wearable robotic technologies on motor recovery and about other therapies that can be combined with exoskeletons to improve their therapeutic effects.
\end{abstract}

Keywords: Exoskeleton, Upper-limb, Rehabilitation, Stroke, Functional Electrical Stimulation, Virtual Reality, Randomized Controlled Trial

\section{Introduction}

About $80 \%$ of stroke survivors suffer from upper-limb paresis [15], which strongly affects their capability to perform activities of daily life (ADL), decreasing their independence and quality of life. Neuroplasticity is the basic mechanism underlying improvements in functional outcomes after stroke. Rehabilitative interventions should make effective use of neuroplasticity for functional recovery, proposing high-intensity, repetitive, task-specific, interactive and individualized training [13. Technology-supported rehabilitation is emerging as a solution to support therapists in providing such training for a long duration, allowing the participants to progress in task difficulty and to achieve the desired movement, so as to increase their motivation [5]. In the last thirty years, several robotic devices for upper-limb rehabilitation have become commercially available [2] Therapeutic robots can be divided into two categories: end-effector devices (e.g. InMotion Arm Robot, known as MIT-Manus [12]) and exoskeleton-type devices 
(e.g. Armeo Power, Hocoma 20]). End-effector devices hold the patients hand or forearm at one point and generate mechanical forces at the interface to move the arm. They are easy to setup but they suffer from limited control of the proximal joints, which could result in abnormal postures. Conversely, exoskeleton-type devices are characterized by robot axes aligned with the anatomical axes of the subject and provide direct control of individual joints, which can minimize abnormal posture or movement and provides a better control of the arm motion [16. These advantages are paid by a more complex design of mechanical structure and control algorithms. This contribution focuses on arm exoskeletons for stroke rehabilitation dealing with mechanical and control design, current evidence about their efficacy, alone or in combination with other therapies, up to potential areas for future research.

\section{Mechanical design and actuation}

Upper limbs anatomical structure is very complex and flexible, permitting the human arm to perform a wide range of movements leveraging on 9 Degrees of Freedom (DOFs). A successfully designed upper-limb exoskeleton operates alongside the human arm smoothly supporting its natural kinematics. In literature, arm exoskeletons mainly provide support at the shoulder and at the elbow, only few of them are provided handles to support the motion of wrist and hand [16. Regardless the number of supported DOFs, the design of upper-limb exoskeletons suggests that robot axes aligned with anatomical axes of the human arm improve robot's wearability and safety. However, since the instantaneous center of rotation of the shoulder changes during motion and since an incorrect axis alignment leads to undesirable interaction forces and uncomfortable postures, additional actuated or passive DOFs have been added to adjust and self-compensate misalignments [11/27/20].

Regarding the actuation mechanisms, three main technologies can be identified: pneumatic, hydraulic and electric actuators. Pneumatic and hydraulic actuators provide higher power to weight ratio with respect to electric motors, but their efficiency strongly depends on pressure losses due to friction or to fluid leaks. Pneumatic and hydraulic, due to their nature, are inherently safe and compliant, thus suitable for applications involving human-robot interaction. These actuators have been widely tested in exoskeletons in the past years, however due to their power consumption, low efficiency and limited control bandwidth, they did not find great consensus [10. On the other side, due to their ability to produce large amount of torque, their commercial availability and their intrinsic precision in controlling motion, current solutions mainly implement stiff joints actuated by electric motors. Nowadays, the interest in compliant control for human-robot interaction has led to the development of new approaches that involve the use of electric motors in series with elastic elements, the so-called series elastic actuators (SEAs) 26]. SEAs were initially developed to improve force control accuracy, meeting interest in the rehabilitation field given provided advantages in terms of safety, tolerance to mechanical shocks and indirect torque 


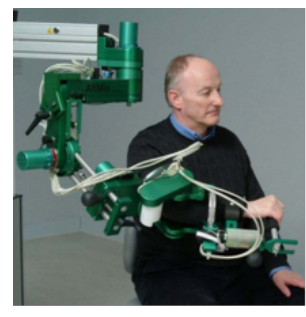

a)

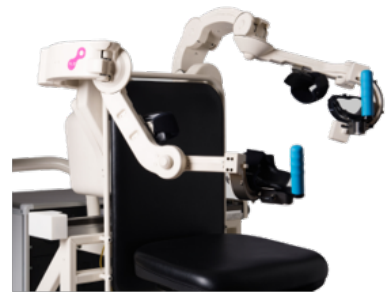

b)

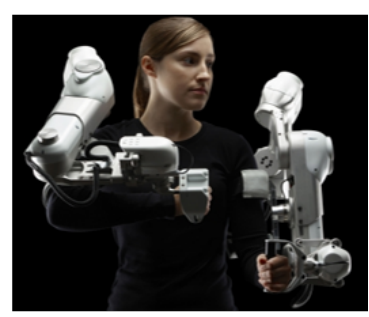

c)

Fig. 1. Upper-limb exoskeletons. a) ARMin III [20] b) AleX [22] c) Harmony [1]

measurements [11. Motors can be both co-located with respect to the axis of rotation of the physiological joints, which leads to a simpler control strategy, but worsens the encumbrance and portability of the system; or positioned behind the exoskeleton, leveraging on the cable-driven approaches, which use wires and pulleys to transmit the power to the actuated joints [22. With the current solutions, however, it is clear that such complex mechanical design has led to powerful, bulky and heavy exoskeletons (Figure 1). This drawback strongly limits their applicability into clinical practice and still requires technological efforts to promote light and wearable exoskeletons for robot-assisted rehabilitation therapy.

\section{Control algorithms}

According to Proietti et al. robot-assisted rehabilitation for upper-limb motor recovery has led to promising results, however most publications do not deal with control aspects [23. Most exoskeletons are controlled in passive or activeassistive mode 22. In passive mode, the robot performs the movement regardless of subject's response to therapy. However, repetitive passive motion of the arm has come out with limited effects on neuroplasticity. Conversely, more complex control strategies based on subject's involvement could lead to better results, at least after the first stages of the rehabilitation process when intensive, repetitive and task-oriented training are the key-features for an effective recovery. This is the case of active-assistive mode, where, after the patient has recovered a sufficient amount of motor control, the controller assists the arm motion in a human-like way, exploiting any residual ability of the patient and letting the user drive the movement, improving patient motivation and self-esteem 23. Many approaches have been proposed to implement assisted-as-needed strategies, but the most widespread rely on admittance control or impedance control, where the controller shapes the dynamical relation between position and force, as a compromise between tracking the position of the end-effector and controlling the effort imposed by the robotic arm [4]. More advanced controllers take advantage 
from friction and gravity compensation techniques to counterbalance the weight and the inertia of the exoskeleton during free-space motion. Other controllers involve the use of surface EMG signals to estimate muscle activation and provide EMG-modulated forces, towards patient's active involvement during exercises [16].

\section{Effects of exoskeletons on upper-limb motor recovery}

The effects of robotic devices on rehabilitative outcomes after stroke have been strongly investigated through randomized controlled trials (RCT) and, in the latest years, through systematic reviews and meta-analysis 291928. Robotic training can be used in addition to usual care to help both therapists and patients in the management of the paralyzed upper-limb and to achieve better rehabilitation outcomes 29. A recent Cochrane review, including 34 trials (1160 participants), demonstrated that stroke patients who receive electromechanical and robot-assisted arm and hand training after stroke might improve their ADL, arm and hand function and strength [19. Meta-analyses of 38 trials (1206 participants) showed significant but small improvements in motor control and muscle strength of the paretic arm and a negative effect on muscle tone when robotic training is compared to usual care 28. Similar results were achieved also in [3], where a comparison between conventional therapy and robot-assisted rehabilitation showed that the latter is more effective in improving upper-limb motor function recovery, especially in chronic stroke patients. It is important to underline that these positive effects on the recovery of arm functions have been mainly achieved using end-effector robotic devices, while there is still a lack of evidence regarding exoskeletons, recently investigated by two systematic reviews 283. They both concluded that so far there is no evidence about the superiority of exoskeleton-assisted training with respect to usual care; however, the effects of exoskeletons have been insufficiently investigated and final conclusion cannot be drawn. Finally, few studies compared different training modalities, but available data indicated that active-assistive mode, stressing the patient's active contribution, led most consistently to improvements in arm function [2].

\section{Combination between exoskeletons and other therapies}

Several therapeutic approaches have been combined with upper-limb exoskeletons, making robotic based-interventions more functional and task-oriented. In

this contribution, we focus on the use of FES and VR in combination with exoskeletons.

\subsection{Functional Electrical Stimulation}

FES has been strongly used to enhance functional recovery of the paretic arm in stroke survivors [8]. When FES is combined with the patient's residual voluntary 
effort [1, cortical plasticity seems to be enhanced, having therefore the potential to improve the therapeutic effects of FES [6]. The combined action of robotassisted therapy supporting elbow and wrist motion and EMG-triggered FES has shown positive effects on muscle coordination in subacute 24 and chronic stroke survivors [25]. FES has been combined also with passive anti-gravity exoskeletons, e.g. ArmeoSpring (Hocoma) 18 or with custom-built passive exoskeleton 21]. In a recent RCT [9, 68 stroke patients were randomized in an experimental group, training with a passive anti-gravity exoskeleton combined with an arm EMG-triggered neuroprosthesis in addition to conventional therapy, and a control group performing only conventional therapy of equal dose. Preliminary results showed the superiority of the training with the hybrid robotic system, supporting the use of exoskeleton devices combined with FES.

\subsection{Virtual Reality}

VR is an interactive and individualized treatment modality, which can provide sensorimotor training in enriched environments, so as to maximize patients engagement [17. Several systematic reviews have shown the superiority of VR training with respect to usual care in improving motor function and ADL independence, mainly when VR is exploited to increase the time spent in therapy 14. Several groups have combined VR with multi-joint upper-limb exoskeletons in order to prevent the slacking effect which may characterize robotic rehabilitation when assistance is too supportive [7. These pilot studies showed promising results in terms of functional improvement and increments of active and passive ranges of motion. In [7, the level of difficulty for the exercise was adjusted by a performance-dependent adaptation algorithm; which may facilitate motor learning by progressively challenging the subject in accordance with the individual capacity. However, more extensive RCTs are needed to demonstrate the superiority of VR combined with upper-limb exoskeletons with respect to VR and robotic rehabilitation alone, as well as to usual care

\section{Perspectives and challenges}

Overall, exoskeleton-type devices have entered the clinical practice of arm stroke rehabilitation. Robotic devices enable several-to-one therapy paradigms, allowing high-dosage and high-intensity treatments, which are recognized as the major positive elements for the recovery process. However, the superiority of exoskeletonassisted training with respect to usual care is still a matter of challenge. To fill this gap, well-design RCTs should be conducted and should include costeffectiveness analysis to evaluate the economical sustainability of these technological solutions. A crucial aspect of exoskeleton design is the human-robot interaction, which should let the human take the lead role, guaranteeing at the same time that the robot closely observe the human and decide when to provide corrective actions. Integration of biological signals, such as EMG, is mandatory 
to achieve this interaction. Despite many significant advances, effective strategies, which can realize minimal assistance paradigms, are still under investigation. Rehabilitation exoskeletons can also be integrated with multiplayer games, which are recently emerged as a promising approach to increase patients motivation. In order to favor the transfer of motor gains to ADL, it is important to integrate distal and proximal arm training; indeed, so far, exoskeleton devices have been focused mainly on the proximal joints but to make robotic training more functional it is mandatory the integration of robotic hand modules. Finally, the development of home-based rehabilitation systems is the ultimate goal for achieving high intensity in post-stroke therapy. Home-based exoskeletons would assure a safe, intensive, and controlled training with limited supervision, under a remote control of a human therapist.

\section{Conflict of interest declaration}

The Authors declare that there is no conflict of interest regarding the publication of this contribution.

\section{References}

1. Ambrosini et al.: A myocontrolled neuroprosthesis integrated with a passive exoskeleton to support upper limb activities. Journal of electromyography and kinesiology : official journal of the International Society of Electrophysiological Kinesiology 24(2) (4 2014) 307-17

2. Basteris et al.: Training modalities in robot-mediated upper limb rehabilitation in stroke: a framework for classification based on a systematic review. Journal of NeuroEngineering and Rehabilitation 11(1) (7 2014) 111

3. Bertani et al.: Effects of robot-assisted upper limb rehabilitation in stroke patients: a systematic review with meta-analysis. Neurological Sciences 38(9) (9 2017) 15611569

4. Calanca et al.: A review of algorithms for compliant control of stiff and fixedcompliance robots. IEEE/ASME Transactions on Mechatronics 21(2) (2016) 613624

5. Chang and Kim: Robot-assisted Therapy in Stroke Rehabilitation. Journal of stroke 15(3) (9 2013) 174-81

6. Gandolla et al.: The Neural Correlates of Long-Term Carryover following Functional Electrical Stimulation for Stroke. Neural Plasticity 2016 (3 2016) 1-13

7. Grimm et al.: Closed-Loop Task Difficulty Adaptation during Virtual Reality Reach-to-Grasp Training Assisted with an Exoskeleton for Stroke Rehabilitation. Frontiers in Neuroscience 10 (11 2016)

8. Howlett et al.: Functional Electrical Stimulation Improves Activity After Stroke: A Systematic Review With Meta-Analysis. Archives of Physical Medicine and Rehabilitation 96(5) (5 2015) 934-943

9. Immick et al.: Hybrid Robotic System for Arm Training After Stroke: Preliminary Results of a Randomized Controlled Trial. In: International Conference on NeuroRehabilitation. (2019) 94-97 
10. Islam et al.: A Brief Review on Robotic Exoskeletons for Upper Extremity Rehabilitation to Find the Gap between Research Porotype and Commercial Type. Advances in Robotics \& Automation 06(03) (11 2018) 1-12

11. Kim and Deshpande: An upper-body rehabilitation exoskeleton Harmony with an anatomical shoulder mechanism: Design, modeling, control, and performance evaluation. International Journal of Robotics Research 36(4) (2017) 414-435

12. Krebs et al.: Robot-Aided Neurorehabilitation: A Robot for Wrist Rehabilitation. IEEE Transactions on Neural Systems and Rehabilitation Engineering 15(3) (9 2007) 327-335

13. Langhorne et al.: Motor recovery after stroke: a systematic review. The Lancet Neurology 8(8) (8 2009) 741-754

14. Laver et al.: Virtual reality for stroke rehabilitation. In Laver, ed.: Cochrane Database of Systematic Reviews. John Wiley \& Sons, Ltd, Chichester, UK (9 2011)

15. Lawrence et al.: Estimates of the prevalence of acute stroke impairments and disability in a multiethnic population. Stroke 32(6) (6 2001) 1279-84

16. Lo and Xie: Exoskeleton robots for upper-limb rehabilitation: State of the art and future prospects. Medical Engineering \& Physics 34(3) (4 2012) 261-268

17. Mazzoleni et al.: Combining Upper Limb Robotic Rehabilitation with Other Therapeutic Approaches after Stroke: Current Status, Rationale, and Challenges. BioMed Research International 2017 (9 2017) 1-11

18. Meadmore et al.: Functional electrical stimulation mediated by iterative learning control and 3D robotics reduces motor impairment in chronic stroke. Journal of neuroengineering and rehabilitation 9 (6 2012) 32

19. Mehrholz et al.: Electromechanical and robot-assisted arm training for improving activities of daily living, arm function, and arm muscle strength after stroke. Cochrane Database of Systematic Reviews (11) (11 2015) CD006876

20. Nef et al.: ARMin III arm therapy exoskeleton with an ergonomic shoulder actuation. Applied Bionics and Biomechanics 6(2) (7 2009) 127-142

21. Pedrocchi et al.: MUNDUS project: MUltimodal Neuroprosthesis for daily Upper limb Support. Journal of NeuroEngineering and Rehabilitation 10(1) (7 2013) 66

22. Pirondini et al.: Evaluation of the effects of the Arm Light Exoskeleton on movement execution and muscle activities: A pilot study on healthy subjects. Journal of NeuroEngineering and Rehabilitation 13(1) (2016) 1-21

23. Proietti et al.: Upper-limb robotic exoskeletons for neurorehabilitation: A review on control strategies. IEEE Reviews in Biomedical Engineering 9 (2016) 4-14

24. Qian et al.: Early Stroke Rehabilitation of the Upper Limb Assisted with an Electromyography-Driven Neuromuscular Electrical Stimulation-Robotic Arm. Frontiers in Neurology 8 (9 2017)

25. Rong et al.: A Neuromuscular Electrical Stimulation (NMES) and robot hybrid system for multi-joint coordinated upper limb rehabilitation after stroke. Journal of NeuroEngineering and Rehabilitation 14(1) (12 2017) 34

26. Sensinger and Weir: Improvements to series elastic actuators. Proceedings of the 2nd IEEE/ASME International Conference on Mechatronic and Embedded Systems and Applications, MESA 2006 (2007)

27. Stienen et al.: Self-Aligning Exoskeleton Axes Through Decoupling of Joint Rotations and Translations. IEEE Transactions on Robotics 25(3) (6 2009) 628-633

28. Veerbeek et al.: Effects of Robot-Assisted Therapy for the Upper Limb After Stroke. Neurorehabilitation and Neural Repair 31(2) (2 2017) 107-121

29. Zhang et al.: Robotic approaches for the rehabilitation of upper limb recovery after stroke. International Journal of Rehabilitation Research 40(1) (3 2017) 19-28 